\title{
Compare and contrast: a cross-national study across UK, USA and Greek experts regarding return of incidental findings from clinical sequencing
}

\author{
Elli G Gourna ${ }^{\star}$, Natalie Armstrong and Susan E Wallace
}

Return of incidental findings (IFs) from clinical sequencing has become a hotly debated topic over the past year. Efforts are being made by several bodies to provide guidance at both national and international levels; however, no studies comparing attitudes of experts across different countries have been published so far. Our goal was to investigate attitudes towards return of IFs from clinical sequencing across UK, USA and Greek experts. Thirty in-depth interviews were conducted with genetics and genomic experts with different backgrounds. Our study revealed more differences when experts were compared according to their professional background than their country. General principles guiding the decision-making and the feedback process were common across all experts but the details of integrating these tests might vary as different professionals reported different needs and attitudes.

European Journal of Human Genetics (2016) 24, 344-349; doi:10.1038/ejhg.2015.132; published online 10 June 2015

\section{INTRODUCTION}

The publication of the US American College of Medical Genetics and Genomics' (ACMG) recommendations in March 2013 ${ }^{1}$ regarding incidental findings (hereafter IFs) from clinical sequencing opened 'Pandora's Box'. The recommendations require participating laboratories to actively search for 56 specified variants every time a patient undergoes clinical sequencing. They also require that these variants, if discovered, should be reported back to the patient (or their parents) regardless of the patient's preferences or age. The wide-ranging discussions around returning IFs, initiated by bioethics experts such as Susan Wolf and focusing on the research setting, ${ }^{2-4}$ have been transferred to the clinical setting with the advent of the use of whole exome or whole genome sequencing (hereafter WE/WGS) to diagnose suspected genetic disorders. ${ }^{5,6}$ Although the ACMG's original recommendations were reviewed and revised a year later (April 2014), ${ }^{7}$ many have been hesitant to adopt them and no consensus yet exists on which results should be reported to patients. As clinical sequencing is expected to be more frequently used in the future, more work is needed to examine the appropriate framework that needs to be created to facilitate the management of IFs. Although we acknowledge the controversy regarding the appropriateness of the term incidental findings' ${ }^{8-10}$ we chose to use it as it was the term used in the original ACMG recommendations (replaced by the term 'secondary findings' in the revised version).

Currently, practical guidance is limited. At an international level, the European Society of Human Genetics (ESHG) encourages the use of targeted tests when possible to avoid IFs and calls for 'prudence' when less targeted tests are used. ${ }^{11,12}$ However, it does not give specifics on the practicalities of returning IFs when these are found. At a national level, the number of countries with any type of guidance remains limited. In the US, apart from the ACMG guidelines, guidance comes from the Presidential Commission for the Study of Bioethics Issues that underlines the need to inform patients in advance about the possibility to discover IFs. ${ }^{13}$ In the UK, the Association of Genetic Nurses and Counsellors (AGNC) and the Public Health Genomics Foundation (PHG) provide some preliminary guidance promoting pre-test counselling and underlining the need to inform patients about the possibility of discovering IFs. ${ }^{14,15}$ The majority of European countries currently lack topic-specific guidance. General principles and current best practices need to be collected and made available, so that individual countries could implement them in accordance with national legislation and cultural norms.

In response to the variety of viewpoints and expert opinions expressed following the publication of the ACMG recommendations, ${ }^{9,16-19}$ we sought other professionals' attitudes regarding the return of findings from clinical sequencing to determine whether such recommendations, or a variation on them, could form the basis of policy for a country with no current guidance. This required comparing attitudes across countries that had differing levels of experience and attendant guidance in dealing with IFs. To this end, we conducted a crossnational comparison across the UK, the USA and Greece, three countries reflecting different approaches to this issue. The USA was chosen as it is at the centre of the current debates and has set a precedent with the ACMG guidelines. The UK was chosen as it represents the example of a country with a long tradition of healthservices' governance but where specific guidance about the return of IFs is currently under development. Finally, Greece was chosen as an example of a country where there is no framework for genetic and genomic tests ${ }^{20}$ and where these technologies are just being introduced. Greece also represents a country that could be looking to other countries, with longer traditions in health governance, for examples on which to base its public health policies. Our intention in this

Department of Health Sciences, University of Leicester, Leicester, UK

*Correspondence: Miss EG Gourna, Department of Health Sciences, University of Leicester, University Road, Adrian Building, Leicester LE1 7RH, UK. Tel: +44 (0)116 252 2267; Fax: +44 (0)116 229 7250; E-mail: eg202@le.ac.uk

Received 13 January 2015; revised 20 April 2015; accepted 24 April 2015; published online 10 June 2015 
comparative work was to determine the elements and issues within the 'return of results' debate that were most key to creating an acceptable process where one did not previously exist. To the best of our knowledge, no studies have yet been published comparing attitudes of experts across different countries on this subject.

\section{METHODS}

Thirty in-depth interviews were conducted with experts from Greece, the UK and the USA. Experts included clinical geneticists (CG), lab-geneticists (LG), genetic counsellors (GC) and experts with a legal and/or bioethical background (LB). For the purpose of this paper all Greek clinicians specialized in genetics will be referred to as clinical geneticists. Additionally, lab-geneticists, genetic counsellors and experts with legal and bioethical background will be classified as non-clinicians. All experts interviewed worked in metropolitan areas, primarily in a clinical setting and/or a university and most of them also conducted research. Their specialties included oncology, neurology and paediatrics. No further individual demographic information is provided in an effort to maintain anonymity - this is a particular issue for Greek participants as the number of genetics experts in that country is limited. Aggregated participant characteristics are presented in Table 1. It should be noted that in Greece there is no recognized specialty of 'genetic counsellor' and as a result, clinical geneticists and lab-geneticists act as genetic counsellors and provide pre and post-test counselling and follow-up.

Recruitment began with initial approaches to established experts (based on their previous experience and published work at a national and international level) and continued with snowball sampling to recruit further participants. Four experts were initially identified from each country and were then asked to suggest colleagues working and publishing in the same area. It should be noted that most of our experts explicitly suggested we contact colleagues who they believed held opposing views as they believed that would be more helpful for our research purposes than suggesting like-minded colleagues. Experts' suggestions were combined with publication searches and background checks to identify the remaining experts.

In total 65 experts were identified and contacted, of whom 36 agreed to be interviewed. Only 30 interviews were actually conducted as scheduling problems meant the remainder could not be arranged within the necessary timeframe. With experts' consent, interviews were audio-recorded and transcribed verbatim. Interviews with Greek experts were conducted in Greek while interviews with UK and US experts were conducted in English. A topic guide with broad areas (see Table 2) was used with all experts to facilitate the discussion and illicit experts' experiences. Interviews typically lasted 30-60 min and were all conducted by one of the authors (EGG).

A constant comparative approach was used for the analysis ${ }^{21,22}$ and NVivo was used to facilitate the process. Initial themes were generated from the research questions that guided but did not constrain the analysis. Themes and sub-themes were developed and iteratively revised. All interviews were analysed by EGG. To maximize the credibility of the analysis and to ensure the quality of the codes produced, NA independently coded several interviews. Notes were compared and the coding framework that was produced was used as the final framework according to which all other interviews were coded.

Table 1 Participants' specialty and country of origin

\begin{tabular}{lccc}
\hline Country of origin & Greece & UK & USA \\
\hline Specialty & & & \\
Clinical geneticist & 3 & 4 & 3 \\
Genetic counsellor & $\left(5^{\mathrm{a}}\right)$ & 3 & 3 \\
Lab-geneticist & 5 & 2 & 1 \\
Legal/ethical specialists & 2 & 2 & 2 \\
\hline
\end{tabular}

${ }^{\mathrm{a} A l l}$ Greek clinicians and two Greek lab-geneticists of our sample reported that they also provide genetic counselling in addition to their other professional obligations.

\section{RESULTS}

After coding all interviews, results were reviewed and synthesized. We concluded after reviewing our results that there were no significant differences found in attitudes when experts were compared based on their country of practice. Only minor differences were found when the topics discussed related to the health-care system in which they worked. Therefore, here we report the significant differences found when responses were compared across the specialties of the experts interviewed, regardless of their country.

\section{Types of results to be returned}

All experts were in agreement that medically actionable results should be given to patients, but there was variation regarding non-medically actionable results. A majority of the clinical geneticists wanted to return only medically actionable results, while genetic counsellors, labgeneticists and experts with legal and ethical background stated that other results should also be reported, such as those that could inform a patient's reproductive choice.

$[P]$ ersonal utility has never been the purpose of medicine. [...] I think the idea of actionability, as the idea to change treatment, the medical actionability, is the way to go with this. This is the way we think in medicine P23USCG

When it concerns a young person and it might affect their reproductive choices you should let him choose, he might want to know. P04GRLG

For non-clinicians, understanding actionability in a strictly medical sense was seen as too narrow as patients and their families could benefit from knowing results for conditions that are not treatable or preventable.

Personal utility. People would want to know a lot of things because that would help them make decisions in their lives!! And that would be actionable for them. If they're going to die soon for example. P18UKGC

\section{Why returning results from WES is a challenge}

There was general agreement among the experts that returning results from WE/WGS differs from information derived from other medical tests and raises significant challenges.

Experts had concerns about returning WES results, the main concern was their ability to understand and interpret genomic data regardless of the increasing scientific knowledge.

I do not think any of us really, really understand what we're in to. I mean when we did the whole genomes, I never really got my head around how big a genome is [...] there's millions of variants in there. The cases where it's been successful, it has been fantastic, but there are still cases where we're having to scratch around trying to find an answer. P15UKCG

For example, because genetic information is shared across the family, this means that feedback and support processes can be particularly difficult, as others, apart from the patient, need to be considered.

[T] he familial decision-making and the familial context is the thing that sets genetics apart from other [medical information]. P12UKGC

Most experts also suggested that genetic data differ because it could lead to potential stigmatization and that is why patients are often reluctant to share their results even within their own family.

We have here a large portion of my patients population that are with the orthodox-Jewish community and there is great stigmatization of having a genetic condition in your family in terms of marriagability and meeting matches and so many of my patients [choose not to receive any results] and they don't tell anyone P22USCG

However, striking differences arose when discussing the rights of the patient and any obligations to family members. The majority of legal/ 
Table 2 Broad topic areas covered during the interviews

\begin{tabular}{|c|c|}
\hline Demographics & $\begin{array}{l}\text { General job description } \\
\text { General experience with clinical sequencing and IFs }\end{array}$ \\
\hline General question & Defining IFs \\
\hline Guidance/Regulation/Support & $\begin{array}{l}\text { Available guidance/regulation in your country and/or your institution } \\
\text { Any other support available for health-care providers }\end{array}$ \\
\hline Previous experiences & $\begin{array}{l}\text { Awareness of the possibility to discover IFs in clinical sequencing } \\
\text { Awareness of the possibility to discover IFs other medical tests } \\
\text { Are the two above topics new or long existing phenomenon } \\
\text { Potential differences between clinical sequencing and other tests and their IFs } \\
\text { Frequency of clinical sequencing in your country, uses, and frequency of discovering IFs } \\
\text { Willingness to use clinical sequencing, potential benefits and concerns } \\
\text { Return of IFs (type of findings to be returned, medically actionable, non-medically actionable etc) } \\
\text { Who makes the decision and who conducts the feedback? } \\
\text { Experiences with patients sharing results with their family }\end{array}$ \\
\hline Future practices & $\begin{array}{l}\text { What type of guidance/regulation/support would you like to have in place? (guidelines/law/other support) } \\
\text { Who should decide about findings that should be returned? } \\
\text { Who should return IFs? } \\
\text { When? }\end{array}$ \\
\hline
\end{tabular}

bioethics experts (five out of seven) suggested that family members might have a legal right to access genetic information especially if results concern life-threatening conditions.

Family members could always claim that they have a legal right to access this information. It is a genetic condition so it affects them all. P09GRLB

On the other hand, the majority of clinical geneticists (nine out of ten) stated that it was more important to protect their patients than be concerned with the needs of the patient's family.

Only the patient. I have no right to inform the family. I have never met them, I don't know them. The patient should decide if and when they want to inform them. P06GRCG

Somewhere in between, all genetic counsellors underlined the need to encourage and support patients to share information with their families.

I think you can't force someone to tell their family about genetics but I think you could encourage people, you give them the tools, the information, advice... and that's as far as you can go here. P25USGC

Different attitudes complicate even further the management of IFs as these suggest that different professionals might manage IFs in a different way thus making the creation of a widely acceptable framework particularly challenging.

Informed consent. There was general agreement across experts that current consent models were inadequate to cover the provision of information from genomic tests.

Right now it is almost impossible to have consent. At least in the traditional way. If we don't know what we might find how can we let them know? And how much time would we need for that?! P05GRLG

Differences were seen when discussing whether there were times when a patient's decision to receive results, or not to receive them, should be respected or whether there were times when that choice should be overridden. Clinical geneticists and genetic counsellors believed that although patients' preferences should be respected, and counselling should support this, some level of paternalism might be unavoidable.

We try very hard not to be directive, not to tell people what to do but you might want to be a bit more persuasive if the condition is very serious or life-threatening. P11UKGC
On the other hand, experts with legal and bioethical backgrounds were more willing to understand patient's autonomy in a more 'active' way and were willing to accept patient's choice to receive results, or not, even if they did not professionally agree with them.

Whoever is doing the genetic counselling should provide all the available information. They should let them know that IFs could be discovered. And then it is the individual's responsibility to ask his doctor if they indeed discovered something. This way we would be sure that the individual actually wants to learn the findings. They need to actively participate! P01GRLB

Once more, the variety observed reflects mostly experts' professional background and does not suggest a difference across the countries.

Targeted vs less-targeted tests. As the majority of experts acknowledged how challenging the use of WES/WGS is and the feedback of IFs, they were asked whether its use should be discouraged and targeted tests remain as the primary diagnostic tool.

Lab-geneticists saw clinical sequencing as a very good source of information about a patient's genetic information (eg, health and other information) and therefore it should be used frequently.

The best thing with it [sequencing] is that you might actually get an answer. Even in cases where you have used everything else [other tests] without any results. P29USLG

Clinical geneticists and genetic counsellors on the other hand were more reluctant to use less targeted tests and were in favour of using targeted tests unless they had no alternative.

Yes and I think that's a general principle of medicine. Don't gather information you don't need. It's a problem. P26USCG

Future actions to enable the integration of WES and WGS into the clinical setting

Experts were asked to describe actions that could help them integrate WES into the clinical setting in the future. Topics discussed included the need to have a clear and comprehensive framework on which guidance could be based; the need to have the most appropriate people be involved in the decision-making and feedback process; and the importance of counselling. 
Future guidance and support. Experts were asked to describe the type of guidance they would like to have. They were given options such as a list that would mandate the exact results that had to be returned (such as the ACMG recommendations) or more general guidelines or recommendations describing the criteria but not restricting the possible results. Although they acknowledged that a list could provide some consistency, it was not the option generally preferred by the experts. Clinical geneticists especially considered that a list would limit their clinical freedom.

Not a list. We should be able to decide what to do according to the family we have in front of us. We are trained to do this and this is actually our job description. We need support not someone depriving us from our clinical discretion. P04GRCG

As mentioned earlier, several experts argued that existing models of informed consent and counselling might not be appropriate for clinical sequencing. They discussed how patients and families should be informed and supported if IFs are to be returned.

Our experts considered that patients do not need very detailed information about genetics but only need to understand the implications that results might have on them and their family. However, our experts differed when discussion how that information should be shared. Clinical geneticists in particular considered that patients need, above everything else, an honest approach and need to trust that their clinicians are acting in their best interest.

I mean I don't think patients are really looking for a detailed $\mathrm{PhD}$ level knowledge of genetics [...] what they're looking for is honesty and openness and an understanding that you're going to do your best to find whatever it is that's causing their condition. P15UKCG

Additionally most experts, especially genetic counsellors (and those acting as genetic counsellors) believed that patients would get a basic understanding if whoever is informing them is patient and willing to spend enough time with them. They also noted that the patient's educational level and lay beliefs should be taken into consideration when deciding what counselling approach to use.

Their level of understanding depends on how much time you spend with them, how patient you are and if you can explain things in their level of understanding. P10GRGC

This difference of opinion will need to be resolved as procedures are developed.

Who should return results? A similar dichotomy was also found when discussing who should return findings. All experts considered that ideally a multidisciplinary team (MDT) should be in charge of the decision and feedback process.

Ideally have a group of people, experiences people in charge. At least one each [from each specialty]. All together they would be able to make a better decision than each one separately. P08GRLG

However, differences were observed when experts were asked to suggest who should return results, if an MDT was not available. Clinical geneticists believed that they should fall to them to return results.

[A]clinician's job has always been to take care of patients. To inform them about good and bad news. We have been trained to do so. We have a much better understanding of the conditions but also of how the human body works. P10GRCG

Contrary, the rest of the experts interviewed said that genetic counsellors were better equipped for this role.

I absolutely think that genetic counsellors are best equipped. There are some very well informed clinicians but clinicians are not always easy to understand. But genetic counsellors that their forte, that's what they do. P28USLB

\section{DISCUSSION}

Our comparison of experts' attitudes towards returning IFs from clinical sequencing showed more similarities than differences, especially concerning the general principles guiding the process. Interestingly, these similarities were consistent across experts and neither their country nor their background seemed to affect their general views. The majority of experts agreed to return medically actionable results and considered the decision making and feedback process particularly challenging; they also agreed that general guidelines are preferred (over a list of specific results); counselling is very important; and that an MDT should ideally be in charge of deciding and returning results.

Differences, however, were observed when experts were compared across professions. The clinical geneticists interviewed here expressed more fears than non-clinicians regarding their understanding of genomic data and their ability to interpret results, while nonclinicians, especially lab-geneticists, expressed more confidence. This may be because actionability can be understood in different ways. ${ }^{23}$ Our clinical geneticists were willing to return only medically actionable results, reflecting their previous experiences and training ${ }^{24-31}$ and confirming findings from other commentators, ${ }^{1,11,27,32,33}$ while our non-clinicians took a broader view of actionability, to include medically actionable results as well as results that might have personal utility. This could reflect the fact that clinicians understand actionability in the strict medical sense and may be uncomfortable with other factors that might unnecessarily add more uncertainty in treatment decisions. If clinical geneticists believed that only IFs that fell within the medically actionable category would be returned, then they would feel comfortable giving them, as it would be little different from their regular medical practice. This contrasts with groups such as genetic counsellors who more commonly deal with patients' social identities, that is, complex personalities with personal, cultural and religious beliefs. ${ }^{34}$ If IFs included those of personal utility, then genetic counsellors would feel comfortable returning those as such discussions fall into their current practices. As a result, 'actionability' is a contested term $^{35}$ that currently provides an unsecure basis on which to formalize policy.

This difference of opinion on actionability continues when discussing who should decide whether IFs should be returned and who should lead that process. Our clinicians believed that they should take on this role if an MDT is not available. Contrary to what has been discussed elsewhere, ${ }^{36}$ our clinicians considered themselves as capable of filling in the gap that an MDT would leave. Interestingly, this also contradicts the doubts expressed earlier about their limited understanding of genetic and genomic data. This contradiction could potentially be explained in two ways. First, the sample of clinicians used for this study consisted of those with a long experience in genetic and genomic tests, which may not be the case for all clinicians, especially for non-specialized clinicians that other studies refer to. ${ }^{36,37}$ Second, this may be because of the different interpretations of actionability as discussed earlier. As Wynn et al. ${ }^{38}$ suggested in a recent publication, clinical training and prior experiences with patients might affect professionals' views on returning IFs. Regardless of whether our clinicians felt they could handle this role, they expressed a preference for having an MDT leading the process, showing that a multi-disciplinary group of professionals might be more appropriate when compared with one person's opinion, even if that person is an expert.

Clinical geneticists in our study were also in favour of the continued use of targeted tests, reflecting past findings ${ }^{12,39,40}$ and again confirming a reliance on current practice. This attitude might change in the near future as new knowledge is acquired regarding benefits of using 
WES and WGS as a diagnostic tool. ${ }^{5}$ However, if this does not happen, a serious concern is raised regarding the limited use of WES and WGS tests. Developing and improving these tests is a questionable investment if clinicians are reluctant to use them because of their reported difficulties in interpreting genomic results. Further training might be required to support clinicians and help them be in a position to fully exploit all available tests (targeted or less-targeted such as WES) and choose the best appropriate for their patient's best interest.

Our experts also acknowledged that current consent practices, where patients are informed of the possible implications resulting from testing, might struggle to cover WES and WGS, an observation that adds to the existing literature about alternative models that might be sought. ${ }^{27,41-46}$ However, our clinicians expressed a preference for broad consent with a 'hint of paternalism' allowing them to make some decisions according to their professional judgment. While reflecting long-term practice, in today's world this raises the possibility of 'a potential conflict between patient autonomy and clinical paternalism'. ${ }^{47}$ Autonomy was discussed by experts here and it appears that is perceived in different ways as has been suggested elsewhere. ${ }^{48,49}$ Clinicians suggested that guidance, in any form it might take, should be topic specific but not too constraining, allowing clinicians and genetic counsellors to act according to their professional judgement when necessary. On the other hand, legal/bioethical experts suggested a more 'active' interpretation of autonomy where patients would have to actively ask for information. Research is needed to explore the appropriateness of traditional informed consent practices and, if needed, investigate possible alternatives. Guidance might have to incorporate the need for new informed consent approaches if research reveals such a gap and to identify that different stakeholders' attitudes will have to be taken into account.

Our study showed more similarities than differences across countries, with most similarities reflecting our experts' professional background and previous experiences. Different notions of actionability, and the IFs that fall into that category, divided our experts, reflecting the differences in their professional practices. This finding leads us to suggest that the development of any guidance for experts who might be involved in returning IFs should take into account any fundamental differences in professional beliefs and practices. This might require creating different guidance for the different specialties, as is currently done for other practice issues. More specifically, decisions must be taken on what constitutes actionability. It could be that instead of one standard procedure for returning IFs, the procedure might need to change depending on what the IF is.

We acknowledge our sample size is relatively small and, as such, our findings should be treated with caution. It is possible, for example, that if we had larger numbers of participants within each cell of Table 1, other factors (eg, type of patient, working environment) may have been revealed as important as professional practice in explaining the different views expressed. However, to the best of our knowledge, this is the first comparative study across different countries on this issue and hence our findings demonstrate aspects of the decisionmaking and feedback process that add important insights into the debates and preparation of guidance that are currently on-going. But further research is needed to confirm our finding that professional background is the basis of fundamental differences between opinions on what constitutes actionability, who should return IFs and how it should be done. This will also require further research with lay people and patients as they may have very different attitudes towards what actionability means for them and from whom or in what setting (eg, from a single clinician or from an MDT) they are happy to receive IFs.

\section{CONFLICT OF INTEREST}

The authors declare no conflict of interest.

\section{ACKNOWLEDGEMENTS}

We would like to thank all the experts from Greece, the UK and the USA for their time and input. Without their insightful comments this work would not have been possible. This study is part of a $\mathrm{PhD}$ program funded by College of Medical, Biological Sciences \& Psychology PhD Studentship, University of Leicester.

1 Green RC, Berg JS, Grody WW et al: ACMG recommendations for reporting of incidental findings in clinical exome and genome sequencing. Genet Med 2013; 15: 565-574.

2 Wolf SM, Paradise J, Caga-anan C: The law of incidental findings in human subjects research: establishing researchers' duties. J Law Med Ethics 2008; 36: 361-383, 214.

3 Wolf SM, Lawrenz Frances P, Nelson CA et al: Managing incidental findings in human subjects research: Analysis and recommendations. J Law Med Ethics 2008; 36: 219-248.

4 Wolf SM: Introduction: the challenge of incidental findings. J Law Med Ethics 2008; 36: 216-218.

5 Yang Y, Muzny D, Xia F et al: Clinical exome sequencing: results from 2000 cases (Abstract \#202). Presented at the 2014 ACMG Annual Clinical Genetics Meeting, March 2014. Nashville, TN, USA.

6 Yang Y, Muzny DM, Reid JG et al: Clinical whole-exome sequencing for the diagnosis of mendelian disorders. N Engl J Med 2013; 369: 1502-1511.

7 ACMG ACMG Updates Recommendation on 'Opt Out' for Genome Sequencing Return of Results. 2014. [Last Accessed 16/06/2014]; Available from https://www.acmg.net/ docs/Release_ACMGUpdatesRecommendations_final.pdf.

8 Christenhusz GM, Devriendt K, Dierickx K: Secondary variants - in defense of a more fitting term in the incidental findings debate. Eur J Hum Genet 2013; 21: 1331-1334.

9 Burke W, Matheny Antommaria AH, Bennett R et al: Recommendations for returning genomic incidental findings? We need to talk! Genet Med 2013; 15: 854-859.

10 Shkedi-Rafid S, Dheensa S, Crawford G, Fenwick A, Lucassen A: Defining and managing incidental findings in genetic and genomic practice. J Med Genet 2014; 51: 715-723.

11 van El CG, Cornel MC, Borry P et al: Whole-genome sequencing in health care. Recommendations of the European Society of Human Genetics. Eur J Hum Genet 2013; 21: S1-S5.

12 van El CG, Dondorp WJ, de Wert G MWR, Cornel MC: Call for prudence in wholegenome testing. Science 2013; 341: 958-959.

13 BioethicsGov Presidential Commission for the Study of Bioethical Issues: ANTICIPATE and COMMUNICATE Ethical Management of Incidental and Secondary Findings in the Clinical, Research, and Direct-to-Consumer Contexts. Washington, DC: Presidential Commission for the Study of Bioethical Issues. 2013.

14 AGNC Association of Genetic Nurses and Counsellors. 2014. [Last Accessed 11/06/ 2014]; Available from http://www.agnc.org.uk/.

15 Hall A, Hallowell N, Zimmern R: Managing Incidental and Pertinent Findings from WGS in the 100,000 Genomes Project. Cambridge: PHG Foundation. 2013.

16 Green RC, Berg JS, Berry GT et al: Exploring concordance and discordance for return of incidental findings from clinical sequencing. Genet Med 2012; 14: 405-410.

17 Jarvik GP, Amendola LM, Berg JS et al: Return of genomic results to research participants: the floor, the ceiling, and the choices in between. Am J Hum Genet 2014; 94: 818-826.

18 Biesecker LG, Green RC: Diagnostic clinical genome and exome sequencing. N Eng/ J Med 2014; 370: 2418-2425.

19 Clayton EW, McCullough LB, Biesecker LG et al: Addressing the ethical challenges in genetic testing and sequencing of children. Am J Bioeth 2014; 14: 3-9.

20 Gourna EG, Armstrong N, Wallace SE: Incidental findings from clinical sequencing in Greece: reporting experts' attitudes. J Commun Genet 2014; 5: 383-393.

21 Pidgeon N, Henwood K: Grounded theory; Hardy M, Bryman A: Handbook of Data Analysis. SAGE: London, 2004

22 Bryman A: Social Research Methods. 4th edn Oxford: Oxford University Press. 2012.

23 Lemke AA, Bick D, Dimmock D, Simpson P, Veith R: Perspectives of clinical genetics professionals toward genome sequencing and incidental findings: a survey study. Clin Genet 2013; 84: 230-236.

24 Biesecker BB, Peay HL: Genomic sequencing for psychiatric disorders: promise and challenge. Int J Neuropsychopharmacol 2013; 1-6.

25 Biesecker LG: Opportunities and challenges for the integration of massively parallel genomic sequencing into clinical practice: lessons from the ClinSeq project. Genet Med 2012; 14: 393-398.

26 Berg JS, Khoury MJ, Evans JP: Deploying whole genome sequencing in clinical practice and public health: meeting the challenge one bin at a time. Genet Med 2011; 13: 499-504.

27 Downing NR, Williams JK, Daack-Hirsch S, Driessnack M, Simon CM: Genetics specialists' perspectives on disclosure of genomic incidental findings in the clinical setting. Patient Educ Couns 2013; 90: 133-138. 
28 Townsend A, Adam S, Birch PH, Lohn Z, Rousseau F, Friedman JM: 'I want to know what's in Pandora's Box': comparing stakeholder perspectives on incidental findings in clinical whole genomic sequencing. Am J Med Genet A 2012; 158A: 2519-2525.

29 Storm C, Agarwal R, Offit K: Ethical and legal implications of cancer genetic testing: do physicians have a duty to warn patients' relatives about possible genetic risks? J Oncol Pract 2008; 4: 229-230.

30 Clarke A, Richards M, Kerzin-Storrar L et al: Genetic professionals' reports of nondisclosure of genetic risk information within families. Eur J Hum Genet 2005; 13: 556-562.

31 Wilson BJ, Forrest K, van Teijlingen ER et al: Family communication about genetic risk: the little that is known. CommunGenet 2004; 7: 15-24.

32 Brandt DS, Shinkunas L, Hillis SL et al: A closer look at the recommended criteria for disclosing genetic results: Perspectives of medical genetic specialists, genomic researchers, and institutional review board chairs. J Genet Couns 2013; 22: 544-553.

33 Krier JB, Green RC: Management of incidental findings in clinical genomic sequencing. Curr Protoc Hum Genet 2013; Chapter 9: Unit 9.23.

34 AGNC Association of Genetic Nurses and Counsellors - Code of Ethics. Association of Genetic Nurses and Counsellors: UK, 2014.

35 Christenhusz GM, Devriendt K, Dierickx K: To tell or not to tell? A systematic review of ethical reflections on incidental findings arising in genetics contexts. Eur J Hum Genet 2013; 21: 248-255.

36 Korf BR, Berry $A B$, Limson $M$ et al: Framework for development of physician competencies in genomic medicine: report of the Competencies Working Group of the Inter-Society Coordinating Committee for Physician Education in Genomics. Genet Med 2014; 16: 804-809.

37 Burton H, Cole T, Lucassen AM: Genomic medicine: challenges and opportunities for physicians. Clin Med 2012; 12: 416-419.
38 Wynn J, Martinez J, Duong J et al: Association of researcher characteristics with views on return of incidental findings from genomic research. J Genet Couns 2015; e-pub ahead of print 17 January 2015.

39 Wolf SM, Annas GJ, Elias S: Point-counterpoint. Patient autonomy and incidental findings in clinical genomics. Science 2013; 340: 1049-1050.

40 McGuire AL, Joffe S, Koenig BA et al: Point-counterpoint. Ethics and genomic incidental findings. Science 2013; 340: 1047-1048.

41 Machini K, Douglas J, Braxton A, Tsipis J, Kramer K: Genetic Counselors' views and experiences with the clinical integration of genome sequencing. J Genet Couns 2014, 23: 496-505.

42 Ormond KE, Cho MK: Translating personalized medicine using new genetic technologies in clinical practice: the ethical issues. Pers Med 2014; 11: 211-222.

43 Kocarnik JM, Fullerton SM: Returning pleiotropic results from genetic testing to patients and research participants. JAMA 2014; 311: 795-796.

44 Jamal Seema M, Joon-Ho Yu, Chong Jessica X et al: Practices and policies of clinical exome sequencing providers: analysis and implications. Am J Med Genet A 2013; 161: 935-950.

45 Netzer C, Klein C, Kohlhase J, Kubisch C: New challenges for informed consent through whole genome array testing. J Med Genet 2009; 46: 495-496.

46 Rigter T, Henneman L, Kristoffersson $U$ et al: Reflecting on earlier experiences with unsolicited findings: points to consider for next-generation sequencing and informed consent in diagnostics. Hum Mutat 2013; 34: 1322-1328.

47 Lohn Z, Adam S, Birch PH, Friedman JM: Incidental findings from clinical genomewide sequencing: a review. J Genet Couns 2013; 23: 463-473.

48 Ross LF, Rothstein MA, Clayton EW: Mandatory extended searches in all genome sequencing: 'incidental findings,' patient autonomy, and shared decision making. JAMA 2013; 310: 367-368.

49 Klitzman R, Appelbaum PS, Chung W: Return of secondary genomic findings vs patient autonomy: implications for medical care. JAMA 2013; 310: 369-370. 